\title{
43. COMMISSION DE PLASMAS ET MAGNETO-HYDRODYNAMIQUE EN ASTROPHYSIQUE
}

President: T.G. Cowling.

SECRETARY: R.J. Tayler.

Report of Meetings, 23 and 28 August 1967

Business Meeting

The Draft Report of the Commission was approved.

The President reported on discussions which he had had about the scope and name of the Commission and the organising committee was asked to look further into this matter, and so bring proposals to the IAU Executive Committee.

On the proposal of L. Davis, Jr., the Commission agreed to express in a resolution the desirability of prompt and regular publication of solar wind data in a similar way to geomagnetic indices. The resolution (drafted by T.G. Cowling, L. Davis, Jr. and R.J. Tayler) was as follows:

If all experimenters studying the solar wind were to pool their data on wind velocities, it would now be possible to produce an almost continuous record, similar, for example, to the record of indices of geomagnetic activity. This would be of great value to a wide range of astronomical and geophysical research.

We therefore ask the International Astronomical Union to express its full support for the prompt and regular publication of solar wind data in one of the journals already publishing data on solar or geomagnetic activity.

P. A. Sturrock reported that a conference, sponsored by the American Astronomical Society and (probably) the American Physical Society, would be held in the United States in the Autumn of 1968 on 'Plasma Instabilities in Astrophysics'. Welcoming such a conference, the Commission agreed to the following motion:

Commission 43 warmly welcomes the proposal to hold a Conference on Plasma Instabilities in Astrophysics under the sponsorship of the American Astronomical Society and the American Physical Society in the early Autumn of 1968 . The Commission regards such a conference as very timely, and would wish to associate itself fully with all steps needed to ensure its success as a truly international conference.

It was proposed by the President and the organising committee that L. Davis, Jr. and R. Lüst be nominated as future President and Vice-President, respectively, of Commission 43, and that the organising committee consist of T.G. Cowling, S. Kaplan and one further member yet to be nominated to succeed $\mathrm{H}$. Alfvén. This was approved.

\section{Scientific Meetings}

\section{August 1967}

The following short papers were presented:

L. Davis, Jr.: Comments on the various theories of the azimuthal components of the motion of the solar wind.

L. Mestel: Magnetic braking and the solar wind.

P. A. Sturrock: A model of the solar wind.

Professor Alfvén also spoke about the role of electric fields in astrophysics. 


\section{August 1967}

The following short papers were presented:

A.B. Severny: 'Comparison of flares and electric currents'.

P. A. Sturrock: 'Theory of solar flares'.

E. Chvojková: 'Some features of particles spiralling along magnetic fields in large gravity fields'. 'Comments on Babcock's theory of the solar magnetic field'.

M. Kopecký: 'New possibility of explaining the sunspot butterfly diagram'.

J.V. Tuominen: 'Comments on solar magnetic field model and possible application to magnetic stars'.

F.G.E. Krause: 'A solution of the dynamo problem by means of a linear theory of M.H.D. turbulence'.

Because of shortage of time the following two papers were unable to be presented:

E. Dobov: 'Similarity relations in magnetohydrodynamics'.

V.V. Vitkevič: 'Some data on an experimental investigation (by methods of radioastronomy) of the velocity and direction of the interplanetary plasma'. 